\title{
Galactic Distribution and Outburst Frequency of Classical Novae
}

\author{
Hilmar W. Duerbeck \\ Astronomisches Institut der Westfälischen Wilhelms-Universität \\ Münster, F.R. Germany
}

\begin{abstract}
The observed rate and spatial distribution of galactic novae can be explained with the concept of two nova populations, the disc novae of faster outburst development, and the slower bulge novae. Similar populations seem to exist in nearby galaxies. The space density of galactic novae depends strongly on the recurrence time, which is unknown and may lie between 500 and 20000 years. If the recurrence time is long, the population of quiescent novae must be identified; the hibernation scenario may or may not be a solution.
\end{abstract}

\section{Sources of nova discoveries}

EASTERN SOURCES. Eastern sources could be extremely important for the derivation of outburst cycle lengths $T$, through matching the positions of bright modern novae with those of historical sightings. The poor positional information of the latter, however, makes this approach very doubtful. Pskovskii $(1971,1972)$ suggested that the nova of 126 AD may be identified with V603 Aql. While the position he adopted differs by only $4^{\circ}$ from the modern position, Stephenson (1976) places the ancient event $26^{\circ}$ away. The object of $\mathrm{AD} 839$, identified by Pskovskii with GK Per, is considered a comet by Stephenson. My own comparison of the positions of nine bright modern novae (V603 Aql, V476 Cyg, V1500 Cyg, DQ Her, CP Lac, V841 Oph, GK Per, CP Pup and RW UMi) with Stephenson's (1976) catalogue yields only one coincidence better than $5^{\circ}$ : the event of AD 891 May 12, with V841 Oph.

The comparison of Stephenson's catalogue with bright candidates in Vogt's (1989) list of quiescent nova candidates also yields no convincing coincidences. Even his two DQ Her-type objects (if the comment "reappeared later" may be interpreted in such a way) have no good counterparts: Stephenson's nova of AD 396 may be identical with OV Tau, or with V471 Tau (Pskovskii 1979), and the nova of $\mathrm{AD} 1431$ with a faint blue variable in Orion; all positions differ, however, by more $\operatorname{than} 10^{\circ}$.

We thus conclude that evidence is scarce that outburst cycles are of the order of 1000 years.

SEMI-PRECISE POSITIONS FROM THE 16TH TO 18TH CENTURY. Here we enter the period of astrometric work by Tycho and his successors. It was fairly easy to identify the two supernova remnants (B Cas = Tycho's SN and V840 Oph = Kepler's SN). Furthermore, two "old" old novae were recovered by Shara et al. $(1985,1986)$, WY Sge (1783) and CK Vul (1670), see below.

VISUAL DURCHMUSTERUNG OBSERVATIONS. The results from a study of the "missing BD stars" (Zinner 1922): W Ari, SU Ari, SY Gem, VZ Gem, SZ Per, NSV00856 Tri, and other visual sightings from the $17^{\text {th }}$ to the $19^{\text {th }}$ centuries are quite frustrating. SU Ari was certainly real because it is recorded independently in the BD and in Chacornac's Atlas Écliptique; a recent observation of Duerbeck's (1984) candidate No.1 with the ESO $3.5 \mathrm{~m}$ NTT revealed that it is a galaxy. For U Leo, Downes and Szkody (1989) found a candidate which shows ellipsoidal light variation with a period of 0.267 days; this can, however, also be produced by a low inclination W UMa system.

I am not aware of studies trying to clarify the status of variable or missing stars observed in the Cordoba and Cape Photographic Durchmusterungen (Thome 1892-1900, Kapteyn 1895 - 1900).

Celestial PhOtography. Only after the widespread use of photography in sky surveying and the simultaneous rise of astrophysics in the late 19th century, had made the stellar universe more 
TABLE 1. Average Number of Novae Discovered per Year

\begin{tabular}{|c|c|}
\hline magnitude interval & nova rate $\left(\mathrm{yr}^{-1}\right)$ \\
\hline$<1$ & 0.04 \\
$1-2$ & 0.02 \\
$2-3$ & 0.04 \\
$3-4$ & 0.03 \\
$4-5$ & 0.05 \\
$5-6$ & 0.14 \\
$6-7$ & 0.47 \\
$7-8$ & 0.58 \\
\hline
\end{tabular}

attractive for professional and amateur astronomers alike, did the number of nova discoveries increase.

Inspection of the temporal development of nova discoveries over the last 100 years, based on the Reference Catalogue of Novae (Duerbeck 1987) and supplementary data, shows that the rate remained fairly constant in the brighter magnitude bins, while it increased for the fainter ones since the 1940s (Fig. 1). Compensating for man-made gaps (in the 1950s the Harvard Sky Patrol was suspended and the Japanese amateurs had not yet entered the scene), the mean nova rate is derived, given in Table I.

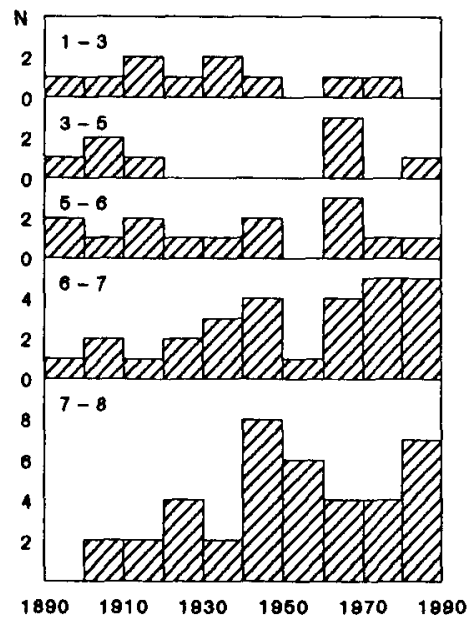

Fig. 1. Number of novae discovered per decade in different bins of apparent magnitude at maximum (sometimes slightly extrapolated towards brighter magnitudes on the basis of available observational material).

\section{Galactic Distribution - Distribution in Galaxies}

The above results, in spite of some incompleteness, can be compared with predictions from a model galaxy. The surface density of novae is derived from $\Sigma=2 \rho_{0} z_{0}=9.5 \cdot 10^{-8} \mathrm{pc}^{-2} \mathrm{yr}^{-1}$, with $\rho_{0}$ and $z_{0}$ taken from Duerbeck (1984). We adopt a mean maximum brightness of $M=-7.5$, and, since a disc population is assumed, a mean galactic obscuration of $1^{\mathrm{m}} \mathrm{kpc}^{-1}$. Fig. 2 shows the comparison between observed and calculated total number counts $N(m)$. 


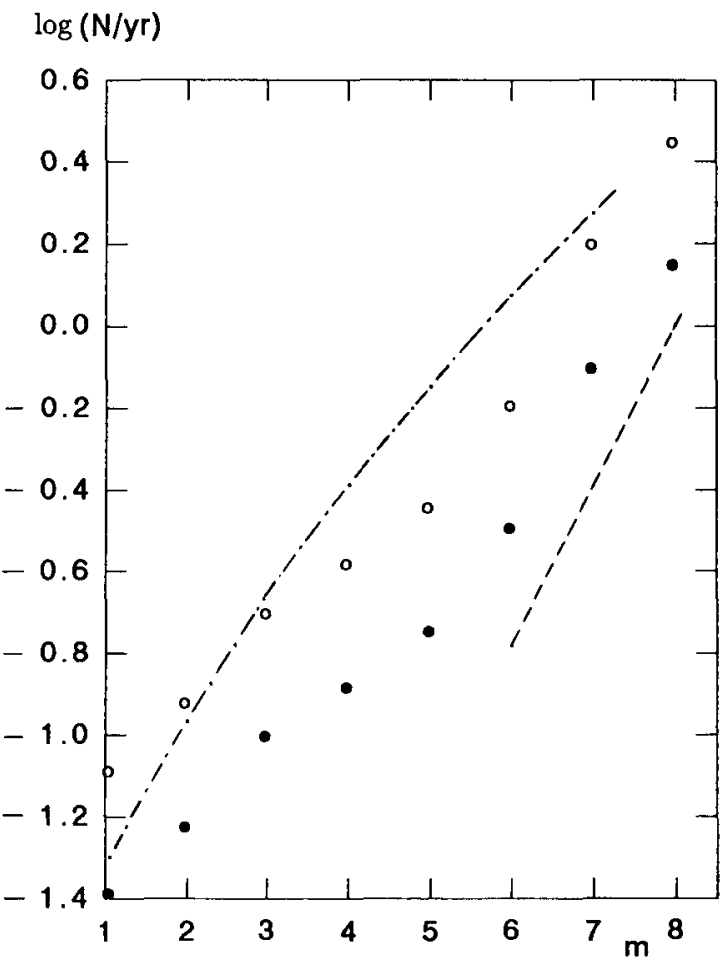

Fig. 2. Theoretical and observed number counts of novae. Filled circles, observed rate; open circles, two times observed rate (i.e. taking into account seasonal changes of nova discoveries); dashed-dotted line: calculated number counts for a disc population; dashed line: expected slope for a bulge population. The observed curves indicate that down to $6^{\mathrm{m}}$, we observe mostly disc novae, where increasingly more novae are missed as we proceed towards fainter magnitudes, while the bulge novae prevail at faint magnitudes.

The distribution of novae in galactic longitude is shown in Fig.3, separately for the brighter and fainter ones.

The following conclusions can be drawn:

- Observed and calculated total number counts coincide reasonably well in the range $0^{\mathrm{m}}$ and $3^{\mathrm{m}}$, as expected, because the space density which was used for the calculation is derived from novae in this magnitude interval (or space volume).

- In the magnitude interval $4^{\mathrm{m}}-6^{\mathrm{m}}$, the observed numbers increase less than predicted by the disc model - it seems that we miss a number of objects (Allen 1954, Schmidt-Kaler 1957).

- From $6^{m}$ onward, the numbers increase more than predicted, in spite of the above effect. The increase seems to follow the $N(m) \propto 10^{0.6 m}$-law. This indicates that we see another population which cannot be attributed to the disc.

- In galactic longitude, an even spread is found for the "near" novae $\left(0^{m}-5^{m}\right)$. Their radial distribution at distances between 7.5 and $9 \mathrm{kpc}$ from the galactic centre is compatible with the radial decline of disc stars in Bahcall and Soneira's model of the Galaxy (Bahcall 1986). The "far" $\left(6^{\mathrm{m}}-8^{\mathrm{m}}\right)$ group is strongly concentrated towards the galactic center, and must belong to the galactic bulge. These objects show a "gap" in Sco-Ara, possibly due to incomplete search (or inaccessibility from Japan). 


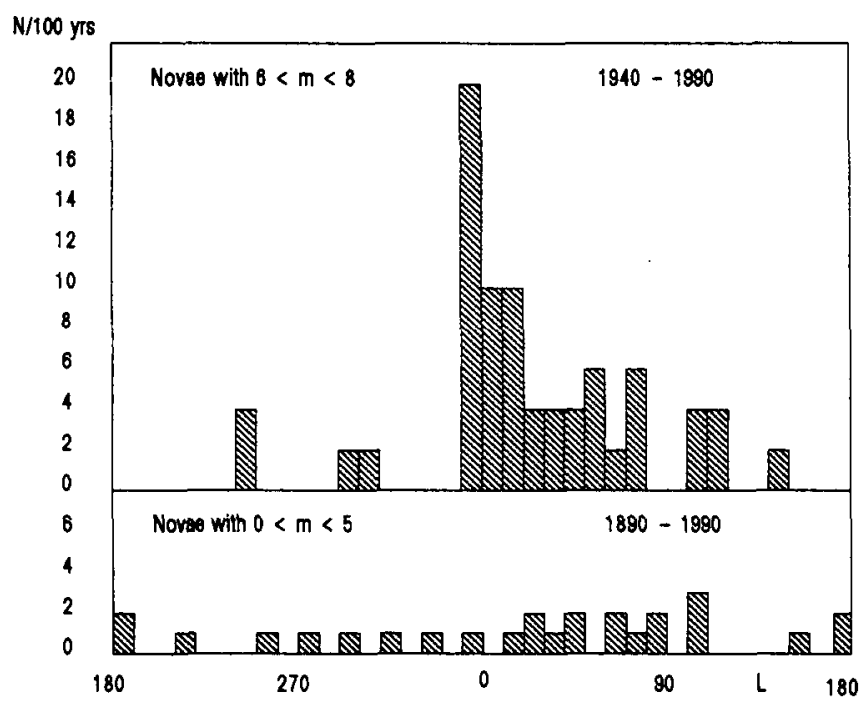

Fig. 3. The distribution of novae in galactic longitude. While the brighter disc novae in the solar neighbourhood are evenly distributed, the fainter novae show a marked concentration towards the galactic center. The asymmetry between the longitude intervals $0^{\circ}-270^{\circ}$ and $0^{\circ}-90^{\circ}$, which is seen in both groups, can be explained by the more thorough and continuous monitoring of the northern sky - at least in the past.

We thus postulate the existence of two populations of novae:

- Disc novae observed in the solar neighbourhood, a fairly young population with mean $z$ values of 125 pc (Duerbeck 1984) or 150 pc (Patterson 1984) - and, as shown below, a generally faster outburst development.

- Bulge novae, concentrated towards the galactic centre, with a larger scale height of about $500 \mathrm{pc}$, and a generally slower outburst development.

The concept of two nova populations also removes the discrepancy of findings for the Galaxy and M31: In the Sb galaxy M31, mainly the novae in the pronounced bulge are observed. In the Galaxy of Sb-c type, the bright novae in the solar neighbourhood belong almost exclusively to the disc, and the fainter novae of the bulge are subject to severe observational selection (interstellar extinction etc.) The nova population of the Large Magellanic Cloud consists almost exclusively of disc novae - very fast or fast objects.

A comparison of percentage contributions of speed classes and light curve types of novae in different stellar systems - galactic bulge, galactic disc, M31 and LMC - is given in Tables II and III, respectively. Despite the fact that the percentages of galactic bulge and M31 novae differ, which can be explained by the novae missed in the galactic bulge, a definitive tendency towards slower speed classes is indicated, suggesting low WD masses and older stellar populations.

Two words of warning: The absolute magnitude at maximum $-t_{3}$-time relation (MMRD-relation) may be different in the two nova populations. Caution has already been voiced in the MMRDcalibration by Duerbeck (1981), and the complaints of Capaccioli et al. (1989), that the MMRDrelation of Cohen (1985), derived for novae in the solar vicinity, yields a poor fit when applied to M31 novae, may result from such a discrepancy.

With the concept of two populations in the Galaxy, all derivations of "the" galactic nova outburst rate have to be considered as uncertain (Sharov 1972, Liller and Mayer 1987, van den Bergh 1988). 
TABLE II. Speed Classes of Novae in Different Stellar Systems

\begin{tabular}{|c|c|c|c|c|}
\hline location & $\mathrm{VF}(<10)$ & $\mathrm{F}(11-25)$ & $\mathrm{MF}(26-80)$ & $\mathrm{S}(81-150)$ \\
\hline disc & $38 \%$ & $33 \%$ & $15 \%$ & $5 \%$ \\
bulge & $24 \%$ & $30 \%$ & $36 \%$ & $9 \%$ \\
M31 & $17 \%$ & $44 \%$ & $31 \%$ & $7 \%$ \\
LMC & $56 \%$ & $44 \%$ & $0 \%$ & $0 \%$ \\
\hline
\end{tabular}

TABle III. Light Curve Types of Novae in Different Stellar Systems

\begin{tabular}{|c|c|c|c|c|}
\hline location & A & B & C & D \\
\hline disc & $50 \%$ & $25 \%$ & $17 \%$ & $8 \%$ \\
bulge & $30 \%$ & $48 \%$ & $15 \%$ & $6 \%$ \\
M31 & $30 \%$ & $27 \%$ & $25 \%$ & $17 \%$ \\
\hline
\end{tabular}

What is known about novae at large distances from the galactic plane? Is there a distinct halo population? X Ser and RT Ser may be linked to symbiotic stars; SV Ari, T Boo and GR Ori are poorly observed objects lacking spectroscopic verification. T Sco and N Oph 1938, also lack spectrocopic confirmation, their maximum absolute magnitudes, however, are those of normal novae if they are placed at the distances of the globular clusters to which they probably belong NGC 6093 (M80) and NGC 6402 (M14). The novae DO Aql, RR Cha, V693 CrA and RW UMi are spectroscopically confirmed. Nebular ejecta of DO Aql and RW UMi have been observed. The number of objects is too small for the derivation of population characteristics.

\section{Space densities, recurrence times, and hibernation}

Space density is not a directly observable quantity, instead we measure the number of nova outbursts per cubic parsec per year in a given part of the galaxy. True space densities can be derived only when we have a reliable estimate of the mean recurrence time.

Theoretical estimates place the recurrence time $T$ somewhere between several hundreds to several ten thousands of years. Thus we cannot expect to observe the total population of (outbursting) novae in a limited time interval. What are the possibilities of getting an estimate of $T$ ? A given volume of space contains novae with recorded outbursts $(\mathrm{N})$, and novae at quiescence (QN). If we know both space densities, we can determine $T$ from

$$
T=\frac{\rho_{N}+\rho_{Q N}}{\rho_{N}} \times T_{\text {obs }}
$$

where $T_{\text {obs }}$ is the time interval during which the given space volume has been carefully searched for nova explosions. There is certainly a spread in $T$ and our sample is biased in favour of frequently outbursting novae; consequently, $T$ will always be underestimated. This effect is neglected in the first approximation. Unfortunately, only quiescent nova "candidates" (QNC) are known, such as some (or all) novalike variables, some (or all) dwarf novae, and slightly detached close binary systems consisting of a white and a red dwarf (the last two cases apply if we assume mild or strong hibernation of nova systems between outbursts). Except for dwarf novae, the space densities of the candidates are hardly known.

If we assume (Duerbeck 1984) that the nova phenomenon is quasi-stationary, i.e., the mass accreted between outbursts $\dot{M} \cdot T$ is equal to the mass ejected during outburst $M_{\text {shell }}$, the time interval $T$ 
between subsequent outbursts can be estimated. Unfortunately, $\dot{M}$ and $M_{\text {shell }}$ are poorly known, and the calculated outburst interval, $3 \cdot 10^{3}$ years, yields a space density which requires ten times more quiescent novae than observed quiescent nova candidates.

Patterson's (1984) approach is basically the same, and he assumes the same shell mass. From the total mass available per outburst, $0.5 M_{\odot}$, the number of outbursts $\left(N=10^{4}\right)$ is derived through dividing by the shell mass. The lifetime is determined from $\dot{M}$, and the recurrence time is

$$
T=\frac{\text { lifetime }}{\text { number of outbursts }} \approx 1.3 \ldots 2.6 \cdot 10^{4} \text { years }
$$

Patterson noted that about 15 times more quiescent novae than candidates must exist, if, as usual, the same absolute magnitude at minimum is assumed for all candidates.

An independent method is the derivation of space densities and recurrence times from a complete sample (Downes 1986). In his survey volume, Downes found 3 novae with recorded outbursts, from which a a space density of $1.1 \cdot 10^{-7} \mathrm{pc}^{-3}$ is derived. The fourth object, V1315 Aql, which he classifies as a nova, is at best a QNC, which I shall add to the novalike objects. Downes' sample thus includes 5 novalikes, with a space density of $4.6 \cdot 10^{-7} \mathrm{pc}^{-3}$. Using (1) we deduce the recurrence time $T=456$ yr (Downes himself, using a formula by Patterson not applicable in this case, found $T=3.35 \cdot 10^{3} \mathrm{yr}$ ). The inclusion of one dwarf nova found in the survey does not change the result significantly. The low value could possibly be attributed to the missing quiescent novae.

The problem of quiecsent novae is obvious: even if we assume that all novalike objects and dwarf novae are quiescent novae, there are too few objects unless the recurrence time is very short.

\section{Hibernation: Pros and Cons}

Hibernation was invented to reconcile the required large space densities of quiescent novae with the lack of observable quiescent nova candidates at $M_{V} \approx 4$, i.e. the mean absolute magnitude of novae before and after eruption. If one assumes that the nova outburst changes the orbital elements of the system in such a way that mass transfer decreases noticeably within a few hundred years, the system may turn into some type of dwarf nova or even a detached system. Only after subsequent loss of angular momentum, due to magnetic braking or gravitational radiation, the system reaches contact again, mass transfer is resumed, and leads, after an appropriate time interval, to another nova explosion (Shara et al. 1986, Livio 1987; a similar idea had already been voiced by Vogt, 1982). The two "old" old novae show indeed fainter absolute magnitudes than generally found in exnovae.

The hibernation model also helps the theoretical argument that TNR cannot occur under sufficiently degenerate conditions at high accretion rates: the average accretion rate in this model is one or two magnitudes lower than the accretion rate observed in postnovae.

Now, the cons:

It has been shown by Duerbeck (1984) that a lowering of the mean accretion rate, i.e. a lowering of the mean brightness, leads to longer recurrence times, and thus to a still higher space density of quiescent novae. A factor 10 in brightness decrease only gives a reduction factor 2 in the number of objects visible to a given apparent magnitude limit, or: fainter objects mean more objects, and the number visible to a given magnitude limit remains roughly the same. With model A of Prialnik and Shara (1986), outburst cycles become, indeed, as long as $10^{5}$ years and space densities as high as $3.8 \cdot 10^{-5} \mathrm{pc}^{-3}$. This implies that the nearest postnova must be closer than $20 \mathrm{pc}$ and should be found in the Catalogue of Nearby Stars (Gliese and Jahreiss 1979). There is none (but the statistics are poor).

What do the two "testimonies" of hibernation teach us? WY Sge (Shara et al. 1984) has a large amplitude $\left(13^{\mathrm{m}} \cdot 5\right)$, but this is still in the range of outburst amplitudes. CK Vul has an amplitude 
of $\approx 18^{\mathrm{m}}$, a peculiar light curve and an even more peculiar, slowly expanding remnant (Shara et al. 1985). Hardly any of the bright novae of our century is expected to show a remnant like this after 300 years. Some doubt is cast on the classification of CK Vul as a classical nova, especially since other stellar outbursts may mimic novae. Seitter (1987a, 1987b, 1989) has shown that the very slow nova V605 Aql (N Aql 1919), which left an extremely hydrogen-poor, slowly expanding remnant and a hot central star, can be interpreted as final helium-flash on the surface of a planetary nucleus. CK Vul may be a similar object.

Finally, recent results on magnetically guided accretion (Williams 1989), and more accurately determined accretion rates (Shaviv 1989) seem to relax the argument of the high accretion rates.

The case is not settled. Vogt (1989), on the basis of very poor observational material from Duerbeck's (1987) compilation, found a tendency for secular brightness decrease in postnovae. More accurate magnitude surveys of postnovae are underway by Duerbeck, Leibowitz and Vogt, and by Szkody (1989).

\section{Summary and Outlook}

Statistical data available so far permit the following conclusions:

- The galactic distribution of novae is explained by two populations of novae, the faster disc novae and the slower bulge novae.

- Recurrence times of outburst may lie at 500,3000 or 20000 years; in the last two cases, the missing quiescent novae will have to be found.

- Hibernation may solve the problem of missing quiescent novae, the observational basis for this model, however, is still too weak.

In order to improve our knowledge of the distribution, properties, and evolution of novae, the following problems should be tackled:

- Better statistical data on bulge novae are needed before novae can be used as standard candles. A powerful observational approach requires wide angle sky patrol in the direction of the Galactic centre, and use of medium- to large size telescopes for spectroscopic studies.

- For proving or disproving hibernation, good statistical data are needed on the secular brightness evolution of classical novae after outburst (surveys are under way). Rotational velocities and UV excesses of late type (G-M) dwarf stars must be measured to decide whether some of them are members of close binary systems with a (hot) companion. A closer look at novalike objects will show whether they resemble quiescent novae sufficiently well.

ACKNOWLEDGEMENTS: The outline of this paper was written when I wore the Visitor's badge of the ESA-Villafranca Satellite Tracking Station, and I thank for the hospitality. The paper was completed when I was Senior Visitor at ESO-La Silla, and I acknowledge the support from the European Southern Observatory.

\section{References:}

Allen, C.W., 1954. Mon. Not. R. astr. Soc., 114, 387.

Bahcall, J.N., 1986. Ann. Rev. Astr. Astrophys., 24, 577.

Bath, G.T., Shaviv, G., 1978. Mon. Not. R. astr. Soc, 183, 515.

Capaccioli, M., Della Valle, M., D'Onofrio, M., Rosino, L., 1989. Astr. J., 97, 1622.

Cohen, J.G., 1985. Astrophys. J., 292, 90. 
Downes, R.A., 1986. Astrophys. J., 307, 170.

Downes, R.A., Szkody, P., 1989. Astr. J., 97, 1729.

Duerbeck, H.W., 1981. Publ. Astr. Soc. Pacific, 93, 165.

Duerbeck, H.W., 1984. Astrophys. Space Sci., 99, 363.

Duerbeck, H.W., 1985. Mitt. Astr. Ges., 63, 190.

Duerbeck, H.W., 1987. A Reference Catalogue and Atlas of Galactic Novae, Reidel, Dordrecht = Space Sci. Rev. 45, 1.

Gliese, W., Jahreiss, H., 1979. Astr. Astrophys. Suppl., 38, 423.

Kapteyn, J.C., 1895 - 1900. Cape Photographic Durchmusterung, I-III, London and Edinburgh.

Liller, W., Mayer, B., 1987. Publ. Astr. Soc. Pacific, 99, 606.

Livio, M., 1987. Comm. Astrophys., 12, 87.

Patterson, J., 1984. Astrophys. J. Suppl., 54, 443.

Prialnik, D., Shara, M.M., 1986. Astrophys. J., 311, 172.

Pskovskii, Yu.P., 1971. Astr. Tsirk., No. 606, 5.

Pskovskii, Yu.P., 1972. Soviet Astr., 16, 23.

Pskovskii, Yu.P., 1979. Sov. Astr. Lett., 5, 209.

Schmidt-Kaler, Th., 1957. Z. Astrophys., 41, 182.

Seitter, W.C., 1987a. Sterne, 63, 44.

Seitter, W.C., 1987b. ESO Messenger, No. 50, 14.

Seitter, W.C., 1989. In Planetary Nebulae, IAU Symp. 131, ed. S. Torres-Peimbert, Kluwer, Dordrecht, p. 315.

Shara, M.M., Moffat, A.F.J., McGraw, T.J., Dearborn, D.S., Bond, H.E., Kemper, E., Lamontagne, R., 1984. Astrophys. J., 292, 763.

Shara, M.M., Moffat, A.F.J., Webbink, R.F., 1985. Astrophys. J., 294, 271.

Shara, M.M., Livio, M., Moffat, A.F.M., Orio, M., 1986. Astrophys. J., 311, 163.

Sharov, A.S., 1972. Sov. Astr., 16, 41.

Shaviv, G., 1989. These Proceedings.

Stephenson, F.R., 1976. Q. Jl. R. astr. Soc., 17, 121.

Szkody, P., 1989. Private Communication.

Thome, J.M., 1892-1900. Resultados del Observatorio Nacional Argentino, XVI-XVIII, Buenos Aires. van den Bergh, S., 1988. Comm. Astrophys., 12, 131.

Vogt, N., 1982. Mitt. Astr. Ges., 57, 79.

Vogt, N., 1989a. In Classical Novae, eds. M.F. Bode, A. Evans, Wiley, Chichester, p. 225.

Vogt, N., 1989b. Astrophys. J. (in press)

Williams, R.E., 1989. Astr. J., 97, 1752.

Zinner, E., 1922. Ergänzungshefte Astr. Nachr., 4, No. 2. 